\title{
A least square solution applied to plate analyses using the traction boundary integral equation
}

\author{
L. Palermo Jr. \\ Faculty of Civil Engineering, Architecture and Urban Design, \\ State University of Campinas, Brazil
}

\begin{abstract}
The least square method was used to solve an overdetermined system of equations in a boundary element formulation, which had the number of integral equations greater than the number of nodes. The solution with the least square method was so good that a regular solution would present similar accuracy only if a fine mesh was used (i.e. double the number of nodes.)The analysis considered the traction boundary integral equation (BIE) to solve a plate bending problem instead of the displacement BIE. Two formulations for traction BIEs were considered, which had the strong singularity reduced with the tangential differential operator (TDO). The strong singularity was reduced in the first formulation without changing other fundamental solution kernels. In the second formulation, the TDO was applied to all fundamental solution kernels involving the multiplication of generalized displacements to reduce the singularities, and the resulting kernels were combinations of those from the displacement BIE.

Keywords: least square solution, traction boundary integral equation, plate bending, Reissner's model, Mindlin's model.
\end{abstract}

\section{Introduction}

Traction BIEs are important when an additional boundary equation is necessary in some problems that do not degenerate solutions using displacement BIEs, like those used in fracture mechanic problems. Strong singularities appear in the fundamental solution kernels of traction BIEs and in stress BIEs at boundary points, which are intrinsically related. The collocation point positions, which are internal points in the boundary element, and the strategy for treating improper integrals are the essential features studied in numerical implementations for 
traction BIEs in plate bending or elasticity problems [1,2]. Strong singularities in the fundamental solution kernels can be reduced by applying the tangential differential operator (TDO) in conjunction with integration by parts, when Kelvin-type fundamental solutions are used. Kupradze first presented an application using the TDO [3] and Balas and co-workers presented some applications of the TDO in boundary element formulations [4]. Regularized boundary element formulations employing TDO for potential and elasticity problems, including those for fracture mechanics problems, were presented by Bonnet [5]. Plane problems of linear fracture mechanics were analyzed with the dual boundary element method using the TDO in the traction BIE [6] and a traction BIE formulation with TDO for meshes using non-conformal interpolations in three dimensional problems was proposed [7].

A formulation for traction BIE in plate bending, considering the shear deformation effect, using the TDO in conjunction with the integration by parts was proposed [8]. The existence of two tensors in the plate bending equilibrium produces a second formulation for the traction BIE [9]. In the first formulation, the strong singularity was reduced whereas the other kernels remained unchanged. In the second formulation, all fundamental solution kernels multiplying generalized displacements had their singularities reduced with the TDO, and the resulting kernels were combinations of those from the displacement BIEs. In spite of the fact that the solutions in the boundary element method usually employ the displacement BIE, plate bending problems were solved with both traction BIEs with the TDO for a better evaluation of their behavior [9]. Similar results were obtained in both formulations, which had isoparametric linear elements in the numerical implementation and employed one collocation point per element in conformal interpolations or two points per element in non-conformal interpolations. The number of collocation points in each element was defined in the code according to the last node condition, i.e. elements with discontinuity at the first node had one collocation point. This feature was necessary to avoid the solution of an overdetermined linear system of equations.

The purpose of the present study is an evaluation of the behavior of both formulations for traction BIE with TDO when the number of collocation points in each element is always equal to the number of nodes regardless of the interpolation type adopted. In spite of the increase in the number of equations, the collocation point positions on each element become always symmetric with benefits to the symmetry of the results obtained, as previously discussed [9]. This strategy produces an overdetermined system of equations when a conformal interpolation is introduced in a small region. The least square solution can be used to solve the overdetermined system of equations. The matrix obtained from the least square solution becomes symmetric and positive definite as an additional benefit. A plate bending problem is employed in the present study to perform the evaluation of the described strategy using the least square solution. 


\section{Traction boundary integral equations with the tangential differential operator}

The equilibrium equations for an infinitesimal plate element under a transverse distributed loading $\mathrm{q}\left(\mathrm{x}_{\mathrm{i}}\right)$ are first written with Latin indices considering the values $\{1,2$ and 3$\}$ and Greek indices considering the values $\{1,2\}$ :

$$
\begin{gathered}
M_{\alpha \beta, \beta}-Q_{\alpha}=0 \\
Q_{\alpha, \alpha}+q=0
\end{gathered}
$$

A unified notation for the Reissner and Mindlin models [8, 9] is used in the following constitutive relations:

$$
\begin{gathered}
M_{\alpha \beta}=D \frac{(1-v)}{2}\left(\psi_{\alpha, \beta}+\psi_{\beta, \alpha}+\frac{2 v}{1-v} \psi_{\gamma, \gamma} \delta_{\mathrm{a} \beta}\right)+\delta_{\alpha \beta} q R E \\
Q_{\alpha}=D \frac{(1-v)}{2} \lambda^{2}\left(\psi_{\alpha}+w_{, \alpha}\right)
\end{gathered}
$$

with

$$
\lambda^{2}=12 \frac{\kappa^{2}}{h^{2}} ; \quad R E=\frac{v}{\lambda^{2}(1-v)}
$$

The plate has a uniform thickness $\mathrm{h}, \mathrm{D}$ is the flexural rigidity, $v$ is the Poisson ratio, $\mathrm{w}$ is the deflection, $\psi_{\alpha}$ is the plate rotation in the direction $\alpha$, and $\delta_{\alpha \beta}$ is the Kronecker delta. The product qRE in Equation (3) corresponds to the linearly weighted average effect of the normal stress component in the thickness direction and should be considered in Reissner's model but not in Mindlin's model, in which it should be considered to be null. The shear parameter $\kappa^{2}$ is equal to $5 / 6$ and $\pi^{2} / 12$ for the Reissner and Mindlin models, respectively.

A unified displacement BIE for the Reissner and Mindlin models can be written in terms of the generalized displacements and tractions presented in [10]:

$$
\begin{aligned}
c_{i j}\left(x^{\prime}\right) u_{j}\left(x^{\prime}\right) & +\int_{\Gamma}\left[T_{i j}\left(x^{\prime}, x\right) u_{j}(x)-U_{i j}\left(x^{\prime}, x\right) t_{j}(x)\right] d \Gamma(x) \\
& =\iint_{\Omega}\left[U_{i 3}\left(x^{\prime}, X\right)-U_{i \alpha, \alpha}\left(x^{\prime}, X\right) R E\right] q(X) d \Omega(X)
\end{aligned}
$$

where $\mathrm{u}_{\alpha}$ equals $\psi_{\alpha}, \mathrm{u}_{3}$ equals $\mathrm{w}, \mathrm{t}_{\beta}$ is the product $\mathrm{M}_{\alpha \beta} \cdot \mathrm{n}_{\alpha}$, and $\mathrm{t}_{3}$ is the product $\mathrm{Q}_{\alpha} \cdot \mathrm{n}_{\alpha} \cdot \mathrm{U}_{\mathrm{ij}}$ represents the rotation $(\mathrm{j}=1,2)$ or the deflection $(\mathrm{j}=3)$ arising from a unit couple $(\mathrm{i}=1,2)$ or a unit point force $(\mathrm{i}=3)$, and $\mathrm{c}_{\mathrm{ij}}$ is an element of the matrix $\mathrm{C}$ related to the collocation point position that makes the diagonal matrix elements equal to one for internal collocation points or equal to 0.5 for 
collocation points on a smooth boundary. The integrand of the domain integral in Equation (7) contains the RE factor, which should be considered null for analyses using the Mindlin model.

The first formulation for traction BIEs, where only the strong singularity is reduced with the TDO $[8,9]$, is given by:

$$
\begin{aligned}
& \frac{1}{2} t_{3}\left(x^{\prime}\right)=n_{\gamma}^{\prime}\left(x^{\prime}\right) D \frac{(1-v)}{2} \lambda^{2}\left(\int _ { \Gamma } \left\{\left[n_{\alpha}(x) M_{3 \alpha \beta . \gamma}\left(x^{\prime}, x\right)-T_{\gamma \beta}\left(x^{\prime}, x\right)\right] u_{\beta}(x)\right.\right. \\
&\left.\left.+Q_{3 \beta}\left(x^{\prime}, x\right) D_{\gamma \beta}\left[u_{3}(x)\right]-T_{\gamma 3}\left(x^{\prime}, x\right) u_{3}(x)\right\} d \Gamma(x)\right) \\
&+n_{\gamma}^{\prime}\left(x^{\prime}\right) \int_{\Gamma}\left[Q_{\beta \gamma}\left(x^{\prime}, x\right) t_{\beta}(x)-Q_{3 \gamma}\left(x^{\prime}, x\right) t_{3}(x)\right] d \Gamma(x) \\
&-n_{\gamma}^{\prime}\left(x^{\prime}\right) \iint_{\Omega} q(X) Q_{3 \gamma}\left(x^{\prime}, X\right) d \Omega(X) \\
& \frac{1}{2} t_{\beta}\left(x^{\prime}\right)=n_{\alpha}^{\prime}\left(x^{\prime}\right) C_{\alpha \beta \rho \gamma}\left(\int _ { \Gamma } \left\{M_{\rho \eta \tau}\left(x^{\prime}, x\right) D_{\gamma \eta}\left[u_{\tau}(x)\right]+n_{\gamma}(x) Q_{\rho \tau}\left(x^{\prime}, x\right) u_{\tau}(x)\right.\right. \\
&\left.\left.+n_{\beta}(x) Q_{\rho \beta, \gamma}\left(x^{\prime}, x\right) u_{3}(x)\right\} d \Gamma(x)\right) \\
&+n_{\alpha}^{\prime}\left(x^{\prime}\right) \iint_{\Gamma}\left[M_{3 \alpha \beta}\left(x^{\prime}, x\right) t_{3}(x)-M_{\gamma \alpha \beta}\left(x^{\prime}, x\right) t_{\gamma}(x)\right] d \Gamma(x) \\
&+n_{\alpha}^{\prime}\left(x^{\prime}\right) \iint_{\Omega} q(X) M_{3 \alpha \beta}\left(x^{\prime}, X\right) d \Omega(X) \\
&+n_{\alpha}^{\prime}\left(x^{\prime}\right) R E \iint_{\Omega} q(X) \frac{\partial}{\partial X_{\gamma}}\left[M_{\gamma \alpha \beta}\left(x^{\prime}, X\right)\right] d \Omega(X) \\
&+\frac{1}{2} R E n_{\beta}^{\prime}\left(x^{\prime}\right) q\left(x^{\prime}\right)
\end{aligned}
$$

$\mathrm{D}_{\mathrm{bm}}($ ) is the tangential differential operator and has the following definition:

$$
D_{b m}[f(y)]=n_{b}(y) f_{, m}(y)-n_{m}(y) f_{, b}(y)
$$

The second formulation for traction BIEs is now written, where all fundamental solution kernels multiplying generalized displacements have their 
singularities reduced with the TDO and the resulting kernels are combinations of those from the displacement BIE, Equation (5), [9]:

$$
\begin{aligned}
\frac{1}{2} t_{3}\left(x^{\prime}\right)=n_{\gamma}^{\prime}\left(x^{\prime}\right) & D \frac{(1-v)}{2} \lambda^{2}\left(\int _ { \Gamma } \left\{M_{3 \alpha \beta}\left(x^{\prime}, x\right) D_{\gamma \alpha}\left[u_{\beta}(x)\right]\right.\right. \\
& +\left[n_{\gamma}(x) Q_{3 \beta}\left(x^{\prime}, x\right)-T_{\gamma \beta}\left(x^{\prime}, x\right)\right] u_{\beta}(x) \\
& \left.\left.+Q_{3 \beta}\left(x^{\prime}, x\right) D_{\gamma \beta}\left[u_{3}(x)\right]-T_{\gamma 3}\left(x^{\prime}, x\right) u_{3}(x)\right\} d \Gamma(x)\right) \\
& +n_{\gamma}^{\prime}\left(x^{\prime}\right) \int_{\Gamma}\left[Q_{\beta \gamma}\left(x^{\prime}, x\right) t_{\beta}(x)-Q_{3 \gamma}\left(x^{\prime}, x\right) t_{3}(x)\right] d \Gamma(x) \\
& -n_{\gamma}^{\prime}\left(x^{\prime}\right) \iint_{\Omega} q(X) Q_{3 \gamma}\left(x^{\prime}, X\right) d \Omega(X) \\
\frac{1}{2} t_{\beta}\left(x^{\prime}\right)=n_{\alpha}^{\prime}\left(x^{\prime}\right) & C_{\alpha \beta \rho \gamma}\left(\int _ { \Gamma } \left\{M_{\rho \eta \tau}\left(x^{\prime}, x\right) D_{\gamma \eta}\left[u_{\tau}(x)\right]+n_{\gamma}(x) Q_{\rho \tau}\left(x^{\prime}, x\right) u_{\tau}(x)\right.\right. \\
& \left.\left.+Q_{\rho \tau}\left(x^{\prime}, x\right) D_{\gamma \tau}\left[u_{3}(x)\right]\right\} d \Gamma(x)\right) \\
& +n_{\alpha}^{\prime}\left(x^{\prime}\right) \iint_{\Gamma}\left[M_{3 \alpha \beta}\left(x^{\prime}, x\right) t_{3}(x)-M_{\gamma \alpha \beta}\left(x^{\prime}, x\right) t_{\gamma}(x)\right] d \Gamma(x) \\
& +n_{\alpha}^{\prime}\left(x^{\prime}\right) \iint_{\Omega} q(X) M_{3 \alpha \beta}\left(x^{\prime}, X\right) d \Omega(X) \\
& +n_{\alpha}^{\prime}\left(x^{\prime}\right) R E \iint_{\Omega} q(X) \frac{\partial}{\partial X_{\gamma}}\left[M_{\gamma \alpha \beta}\left(x^{\prime}, X\right)\right] d \Omega(X) \\
& \frac{1}{2} R E n_{\beta}^{\prime}\left(x^{\prime}\right) q\left(x^{\prime}\right)
\end{aligned}
$$

\section{Numerical implementation}

The generalized displacements and their derivatives were assumed to be continuous along the boundary in the development to obtain the traction BIEs using TDO. Additional terms in Equations (6) to (9) have to be introduced when non-conformal interpolations are used and the corresponding equations are given by [9]: 


$$
\begin{aligned}
& \begin{array}{l}
\frac{1}{2} t_{3}\left(x^{\prime}\right)=(\text { terms on RHS of Equation } 6) \\
\quad+n_{\gamma}^{\prime}\left(x^{\prime}\right) D \frac{(1-v)}{2} \lambda^{2}\left[e_{3 \beta \gamma} Q_{3 \beta}\left(x^{\prime}, x\right) u_{3}(x)\right]_{0}^{\Gamma} \\
\begin{aligned}
\frac{1}{2} t_{\beta}\left(x^{\prime}\right)=(\text { terms on RHS of Equation } 7) \\
+
\end{aligned} \\
\begin{aligned}
\frac{1}{2} t_{3}^{\prime}\left(x^{\prime}\right) C_{\alpha \beta \rho \gamma}\left[e_{3 \eta \gamma} M_{\rho \eta \tau}\left(X^{\prime}, x\right) u_{\tau}(x)\right]_{0}^{\Gamma} \\
+
\end{aligned} \\
+n_{\gamma}^{\prime}\left(x^{\prime}\right) D \frac{(1-v)}{2} \lambda^{2}\left(\left[e_{3 \alpha \gamma} M_{3 \alpha \beta}\left(x^{\prime}, x\right) u_{\beta}(x)\right]_{0}^{\Gamma}\right. \\
\left.+\left[e_{3 \beta \gamma} Q_{3 \beta}\left(x^{\prime}, x\right) u_{3}(x)\right]_{0}^{\Gamma}\right) \\
\begin{aligned}
\frac{1}{2} t_{\beta}\left(x^{\prime}\right)=(\text { terms on } R H S \text { of Equation } 9) \\
+
\end{aligned} \\
+n_{\alpha}^{\prime}\left(x^{\prime}\right) C_{\alpha \beta \rho \gamma}\left(\left[e_{3 \eta \gamma} M_{\rho \eta \tau}\left(x^{\prime}, x\right) u_{\tau}(x)\right]_{0}^{\Gamma}\right. \\
\left.+\left[e_{3 \tau \gamma} Q_{\rho \tau}\left(x^{\prime}, x\right) u_{3}(x)\right]_{0}^{\Gamma}\right)
\end{array}
\end{aligned}
$$

Linear mapping functions were used to represent displacements and efforts in the boundary elements. The same mapping function was used for conformal and non-conformal interpolations with nodal parameters always positioned at ends of the elements.

The continuity for derivatives of displacement functions at the collocation point position is required for traction BIEs, Equations (6) to (9). The collocation points for traction BIEs were shifted to the interior of the element at a distance of a sixth part of its length starting from the end to satisfy the continuity requirement because the nodes were always fixed at the element ends. Two collocation points per element were used regardless of the interpolation type adopted. The collocation points positions ( $\left.\xi^{\prime}\right)$, in the boundary line, were \pm 0.67 , in the range $(-1,1)$, for continuous or discontinuous elements.

Analytical expressions were used to evaluate singular integrals with the Cauchy principal value sense whereas the Gauss-Legendre scheme was used for regular integrals. An expansion for small arguments was considered for terms containing modified Bessel functions with real arguments $\left(\mathrm{K}_{0}, \mathrm{~K}_{1}\right)$ [11]. The diagonal terms were directly obtained using the mapping function and the collocation point position on the element. An overdetermined linear system of equations can be obtained when a conformal interpolation is introduced in a small boundary region because the number of integral equations becomes greater than the number of nodes.

The basic method for solving overdetermined systems using least squares was employed. Given the system A.x equals b, with an $\mathrm{m} \times \mathrm{n}$ matrix A $(\mathrm{m}>\mathrm{n})$, the equation had both sides multiplied by the transpose of $\mathrm{A}$. The normal equations were obtained, i.e. the standard square system of linear equations. It is necessary to point out that any solution of the normal equations is a correct solution to the 
least squares problem, There is a unique solution in the case when $\mathrm{A}^{\mathrm{t}} \mathrm{A}$ is nonsingular and an infinite number of solutions in the case when $A^{t} A$ is singular, and all of them are correct solutions to the least squares problem. The present analysis employed colocation points positioned in admissible places, i.e. collocation points positioned on different places on the boundary, as required for a nonsingular matrix, and a unique solution is obtained. Furthermore, the matrix obtained from $\mathrm{A}^{\mathrm{t}} \mathrm{A}$ is symmetric and positive definite, as defined for this matrix type [13].

\section{Example: the torsion of cube}

This problem was selected because the non-symmetric condition of the loading is better to evaluate the accuracy of the boundary element formulation using traction BIEs. The cube has side length equal to $2 \mathrm{a}$, two opposite faces are free of stress and other faces are under torsion according to the Saint-Venant hypotheses (free warping). The deflection (w) and the plate rotation in the direction $\mathrm{y}\left(\psi_{\mathrm{y}}\right)$ were used to introduce torsion according to the prescribed rotation angle $(\varphi)$ :

$$
\psi_{y}=\varphi a ; \quad w=\varphi a y
$$

Three meshes were used in the complete plate model and six Gauss points in the numerical integration. Two nodes were considered in each corner and conformal interpolations were adopted along the sides for the following meshes: 128 elements (132 nodes), 192 elements (196 nodes) and 256 elements (260 nodes). The results were compared with regular solutions using quadratic elements [1], in which the traction BIE contained the strong singularity and 128 discontinuous elements (384 nodes) were used, as well as using linear elements [9], in which the traction BIEs used the TDO, beyond the Reissner solution [12].

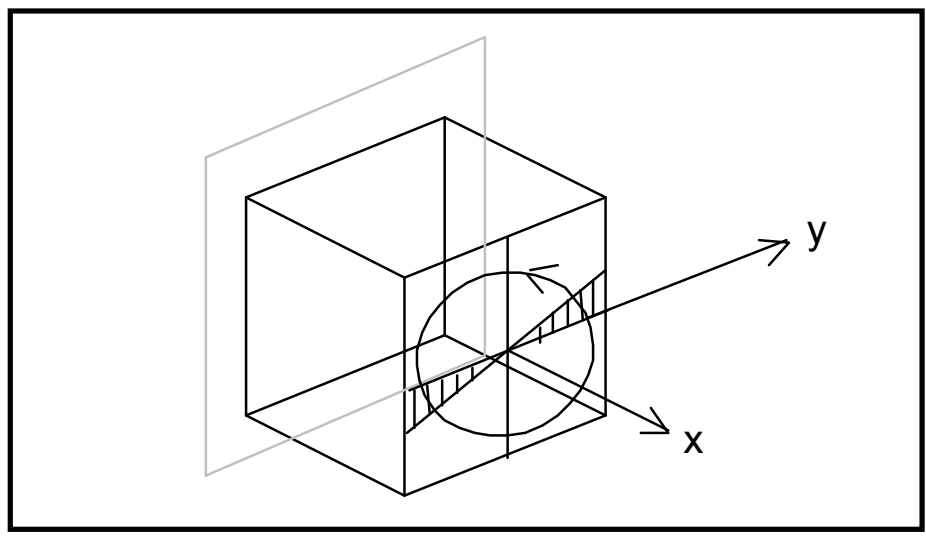

Figure 1: Torsion of a cube. 
The greatest differences to the Reissner solution appeared in the results obtained for plate rotations in the normal direction because these values are indirectly related to the displacements prescribed. These results were used to evaluate the accuracy using the least square solution. The values for distributed shear and twisting moments were not used, because they are directly related to the displacements constrained and the differences to the Reissner solution were extremely low. Values obtained with the first and the second formulation are presented in Table 1 and in Table 2, respectively, where the last two rows show the maximum difference to Reissner's solution and the computation time using a Dell Latitude D510 (Pentium M, Id. 0608, 1.6GHz).

Table 1: $\quad$ Plate rotations in the $\mathrm{x}$ direction along the edge $\left(\frac{\psi_{x}(y)}{\psi_{x}(a)}\right)$ with the first formulation for traction BIE with TDO.

\begin{tabular}{|c|c|c|c|c|c|c|c|c|c|}
\hline \multirow{3}{*}{$\frac{y}{a}$} & \multirow{3}{*}{ [12] } & \multicolumn{2}{|c|}{$\begin{array}{c}\text { Discontinuous } \\
\text { elements }\end{array}$} & \multicolumn{6}{|c|}{ Continuous elements } \\
\hline & & \multirow{2}{*}{$\begin{array}{c}128 \mathrm{QE} \\
384 \mathrm{~N} \\
{[4]}\end{array}$} & \multirow{2}{*}{$\begin{array}{c}128 \mathrm{LE} \\
256 \mathrm{~N} \\
{[9]}\end{array}$} & \multicolumn{2}{|c|}{$\begin{array}{l}128 \mathrm{LE} \\
132 \mathrm{~N} \\
\end{array}$} & \multicolumn{2}{|c|}{$\begin{array}{l}192 \mathrm{LE} \\
196 \mathrm{~N} \\
\end{array}$} & \multicolumn{2}{|c|}{$\begin{array}{l}256 \mathrm{LE} \\
132 \mathrm{~N} \\
\end{array}$} \\
\hline & & & & [9] & L.S.S. & Reg. & L.S.S & Reg. & L.S.S \\
\hline 1.00 & 1.000 & 1.000 & 1.000 & 1.000 & 1,000 & 1,000 & 1,000 & 1,000 & 1,000 \\
\hline 0.75 & -0.055 & -0.051 & -0.058 & -0.056 & $-0,058$ & $-0,055$ & $-0,056$ & $-0,055$ & $-0,056$ \\
\hline 0.50 & -0.387 & -0.382 & -0.389 & -0.387 & $-0,389$ & $-0,386$ & $-0,387$ & $-0,386$ & $-0,387$ \\
\hline 0.25 & -0.292 & -0.290 & -0.294 & -0.292 & $-0,294$ & $-0,291$ & $-0,292$ & $-0,292$ & $-0,292$ \\
\hline 0.00 & 0.000 & 0.000 & 0.000 & 0.000 & 0,000 & 0,001 & 0,000 & 0,000 & 0,000 \\
\hline-0.25 & -0.292 & -0.290 & -0.294 & -0.295 & $-0,294$ & $-0,293$ & $-0,292$ & $-0,293$ & $-0,292$ \\
\hline-0.50 & -0.387 & -0.382 & -0.389 & -0.391 & $-0,389$ & $-0,388$ & $-0,387$ & $-0,387$ & $-0,387$ \\
\hline-0.75 & -0.055 & -0.051 & -0.058 & -0.060 & $-0,058$ & $-0,057$ & $-0,056$ & $-0,056$ & $-0,056$ \\
\hline-1.00 & 1.000 & 1.000 & 0.00 & 1.000 & 1,000 & 1,000 & 1,000 & 1,000 & 1,000 \\
\hline \multicolumn{2}{|c|}{$\begin{array}{c}\text { Maximum } \\
\text { difference (\%) }\end{array}$} & 7.27 & 5.45 & 9.09 & 5,45 & 3.64 & 1.82 & 1.82 & 1.82 \\
\hline \multicolumn{2}{|c|}{$\begin{array}{l}\text { Computation } \\
\text { Time }(\mathrm{sec})\end{array}$} & - & 7.546 & 4.265 & 6.844 & 9.406 & 16.89 & 16.55 & 59,17 \\
\hline
\end{tabular}

$\mathrm{QE}=$ quadratic elements, $\mathrm{LE}=$ linear elements, $\mathrm{N}=$ nodes, Reg. $=$ regular solution

The results obtained from the least square solution (LSS) using 128 and 192 continuous elements (132 and 196 nodes) had similar accuracy and computation time to regular solutions using 128 discontinuous elements (256 nodes) and 256 continuous elements (260 nodes), respectively. In spite of the slightly greater computation time in the LSS with 192 elements than the regular solution using 256 elements, symmetrical results appeared in the LSS. Furthermore, it can be pointed out the regular solution with 256 elements is close to the saturation for 
this problem, as can be noted in the comparison between values obtained from the LSS and the regular solution, which presented low differences.

Table 2: $\quad$ Plate rotations in the $\mathrm{x}$ direction along the edge $\left(\frac{\psi_{x}(y)}{\psi_{x}(a)}\right)$ TDO 2.

\begin{tabular}{|c|c|c|c|c|c|c|c|c|c|}
\hline \multirow{2}{*}{$\frac{3}{a}$} & \multirow{2}{*}{$\begin{array}{c}\text { Discontinuous } \\
\text { elements }\end{array}$} & \multicolumn{6}{|c|}{ Continuous elements } \\
\cline { 3 - 10 } & & $\begin{array}{c}128 \mathrm{QE} \\
384 \mathrm{~N}\end{array}$ & $\begin{array}{c}128 \mathrm{LE} \\
256 \mathrm{~N}\end{array}$ & \multicolumn{2}{|c|}{$128 \mathrm{LE}$} & \multicolumn{2}{|c|}{$192 \mathrm{LE}$} & \multicolumn{2}{c|}{$\begin{array}{c}256 \mathrm{LE} \\
132 \mathrm{~N}\end{array}$} \\
\cline { 3 - 11 } & & {$[4]$} & {$[9]$} & {$[9]$} & L.S.S. & Reg. & L.S.S & Reg. & L.S.S \\
\hline 1.00 & 1.000 & 1.000 & 1.000 & 1.000 & 1,000 & 1,000 & 1,000 & 1,000 & 1,000 \\
\hline 0.75 & -0.055 & -0.051 & -0.059 & -0.057 & $-0,059$ & $-0,056$ & $-0,057$ & $-0,056$ & $-0,056$ \\
\hline 0.50 & -0.387 & -0.382 & -0.391 & -0.389 & $-0,391$ & $-0,388$ & $-0,389$ & $-0,387$ & $-0,388$ \\
\hline 0.25 & -0.292 & -0.290 & -0.295 & -0.293 & $-0,295$ & $-0,293$ & $-0,293$ & $-0,292$ & $-0,293$ \\
\hline 0.00 & 0.000 & 0.000 & 0.000 & 0.000 & 0,000 & 0,001 & 0,000 & 0,000 & 0,000 \\
\hline-0.25 & -0.292 & -0.290 & -0.295 & -0.297 & $-0,295$ & $-0,294$ & $-0,293$ & $-0,293$ & $-0,293$ \\
\hline-0.50 & -0.387 & -0.382 & -0.391 & -0.393 & $-0,391$ & $-0,390$ & $-0,389$ & $-0,388$ & $-0,388$ \\
\hline-0.75 & -0.055 & -0.051 & -0.059 & -0.061 & $-0,059$ & $-0,058$ & $-0,057$ & $-0,056$ & $-0,056$ \\
\hline-1.00 & 1.000 & 1.000 & 1.000 & 1.000 & 1,000 & 1,000 & 1,000 & 1,000 & 1,000 \\
\hline $\begin{array}{r}\text { Maximum } \\
\text { difference (\%) }\end{array}$ & 7.27 & 7.27 & 10.91 & 7.27 & 5.45 & 3.64 & 1.82 & 1.82 \\
\hline $\begin{array}{r}\text { Computation } \\
\text { Time (s) }\end{array}$ & - & 8.094 & 4.656 & 7.422 & 10.14 & 18.01 & 17.91 & 60.55 \\
\hline
\end{tabular}

\section{Conclusions}

The strategy of using the number of collocation points for traction BIEs equal to the number of nodes in each element regardless of the interpolation type adopted, and employing the least square solution, was interesting, according to the results presented for the plate bending problem. Asymmetry issues in conformal interpolations, which have been discussed [9], were fixed in this strategy even using linear elements.

The other benefit with this strategy was hidden in the present analysis because only meshes for plane problems were used, which were simple lines. In three dimensional analyses, the computer algorithm to employ traction BIEs in surface regions using conformal interpolations can be simplified with this technique.

The increase in the computation time due to the use of more collocation points and operations of the least square solution can be justified by the increase of the accuracy without increasing the number of nodes, i.e. degrees of freedom. Furthermore, the benefit of obtaining a symmetric positive definite matrix can be interesting when iterative solutions are required in a problem. Thus, this strategy 
can be suggested even when few traction BIEs are used in conjunction with the displacement BIE that does not degenerate a boundary element solution.

On the other hand, the time to perform matrix operations can be reduced in the computation process when parallel programming instructions are employed in the code.

\section{Acknowledgement}

The author is grateful for CNPq support for the development of studies on plates.

\section{References}

[1] Rashed YF, Aliabadi MH, Brebbia CA. Hypersingular boundary element formulation for Reissner plates. Int J Solids Struct, 35(18), 2229-49, 1998.

[2] Guiggiani, M. Hypersingular formulation for boundary stresses evaluation. Eng. Anal. Bound. Elem., 14, 169-179, 1994.

[3] Kupradze, V.D., Three-dimensional problems of the mathematical theory of elasticity and thermoelasticity. North Holland, 1979.

[4] Balas, J., Sladek, J, Sladek, V., Stress Analysis by Boundary Element Methods, Elsevier Science Publishers, 1989.

[5] Bonnet, M. Boundary Integral Equation Methods for Solids and Fluids, John Wiley and Sons Ltd, 1999.

[6] Palermo Jr., L., Almeida, L.P.C.P.F. and Gonçalves, P.C., The Use of the Tangential Differential Operator in the Dual Boundary Element Equation, Structural Durability \& Health Monitoring, vol.2, no.2, pp. 123-130, Tech Science Press, 2006.

[7] Palermo Jr., L., Almeida, L.P.C.P.F., On the Use of the Tangential Differential Operator in the Traction Boundary Integral Equation of the Dual Boundary Element Method for Three Dimensional Problems, ICCES, vol.7, no.2, pp. 83-87, 2008.

[8] Palermo Jr., L., Tangential differential operator applied to stress and traction boundary integral equations for plate bending including the shear deformation effect, BEM 33, Editors C.A. Brebbia, 2011.

[9] Palermo Jr., L., The tangential differential operator applied to a stress boundary integral equation for plate bending including the shear deformation effect, Eng Anal Bound Elem, 36, 1213-1225, 2012

[10] Weeën, F., Application of the boundary integral equation method to Reissner's plate model, International Journal for Numerical Methods in Engineering, vol. 18, 1-10, 1982.

[11] Abramowitz M, Stegun I. Handbook of mathematical functions, New York: Dover Publications; 1972.

[12] Reissner, E., The Effect of Transverse Shear Deformation on the Bending of Elastic Plates, Journal of Applied Mechanics, 1945.

[13] Birkhoff, G., Mac Lane, S., A Survey of modern algebra, $4^{\text {th }}$. ed., Macmillan Publishing Co. Ltd., 1977. 